\title{
Planetary nebulae as mass tracers in galaxies
}

\author{
Aaron J. Romanowsky \\ Departamento de Física, Universidad de Concepción, Casilla 160-C, Concepción, Chile \\ email: romanow@astro-udec.cl
}

\begin{abstract}
Planetary nebulae are useful kinematic tracers of the stars in all galaxy types. I review recent observationally-driven developments in the study of galaxy mass profiles. These have yielded surprising results on spiral galaxy disk masses and elliptical galaxy halo masses. A key remaining question is the coupling between PNe and the underlying stellar populations.
\end{abstract}

Keywords. planetary nebulae: general, galaxies: elliptical and lenticular, cD, galaxies: spiral, galaxies: kinematics and dynamics, galaxies: halos, dark matter

\section{Introduction}

Planetary nebulae (PNe) are of great importance in galaxy studies as unique observable proxies for stars in regions of low surface brightness. They can be used to infer the spatial, kinematic, and chemical properties of the underlying stellar population, at distances of up to $100 \mathrm{Mpc}$ (cf. 1 Mpc for resolved stellar spectroscopy). Here I review the use of $\mathrm{PN}$ kinematics to probe mass distributions in galaxies, with particular attention to disk masses of spiral galaxies ( $(2)$, and halo masses of elliptical galaxies ( $(3)$. PN kinematics are also useful for studying angular momentum, orbit properties, and substructure in galaxies - topics beyond the scope of this review.

In all such dynamical studies, it is important to know what is the underlying stellar population traced by the observed PNe in external galaxies. These (bright) PNe are frequently assumed to originate with an "old" stellar population that comprises the bulk of the stellar mass in the galaxy. This assumption has a great deal of theoretical uncertainty (e.g., Marigo et al. 2004; Ciardullo et al. 2005; Buzzoni et al. 2006), but empirically, it works remarkably well when PN numbers are compared to stellar photometry. I will return to this issue later, especially in $\S 3.3$. A summary is in $\S 4$.

\section{Planetary nebula kinematics in disk galaxies}

\subsection{The Milky Way}

There are unique pros and cons to studying PNe in the Milky Way vs. extragalactically. Probably the biggest dynamical challenge is the PN distance uncertainty. PN rotation curves have been derived to 14 kpc (e.g., Schneider \& Terzian 1983; Maciel \& Lago 2005), supporting the presence of a dark halo. But it may be more useful to turn the analysis around and use other mass constraints to derive the PN distance scale (Phillips 2001); or to use PNe to study the dynamical structure of the Galaxy (e.g., Beaulieu et al. 2000).

\subsection{Observations in spiral galaxies}

PN kinematics in spiral galaxies outside the Milky Way have not been studied much (relative to elliptical galaxies) because of possible contamination from other emission-line regions, and because of the availability of alternative mass tracers. However, there are some recent developments in this area, based upon observations summarized in Table 1. 
Table 1. Selected observations of planetary nebula velocities in external galaxies

\begin{tabular}{|c|c|c|c|c|c|c|}
\hline Galaxy & Type & $M_{B} \mathrm{I}$ & $\begin{array}{c}\text { Distance } \\
(\mathrm{Mpc})\end{array}$ & pe+Instrument & $\begin{array}{r}\text { Number of } \\
\text { velocities }\end{array}$ & Ref. \\
\hline $\begin{array}{l}\text { Spirals } \\
\text { M } 94 \\
\text { M } 33 \\
\text { M } 31 \\
\text { M } 83\end{array}$ & $\begin{array}{l}\mathrm{Sab} \\
\mathrm{Sc} \\
\mathrm{Sb} \\
\mathrm{SBc}\end{array}$ & $\begin{array}{l}-19.7 \\
-19.0 \\
-21.2 \\
-20.5\end{array}$ & $\begin{array}{l}5 \\
1 \\
1 \\
4\end{array}$ & $\begin{array}{c}\text { WHT+ISIS } \\
\text { WIYN+HYDRA } \\
\text { WHT+WYFFOS, WHT+PN.S } \\
\text { CTIO+HYDRA }\end{array}$ & $\begin{array}{r}67 \\
140 \\
2615 \\
182\end{array}$ & $\begin{array}{c}\mathrm{D}+00 \\
\mathrm{C}+04 \\
\mathrm{H}+06, \mathrm{M}+06 \\
\mathrm{HC} 06\end{array}$ \\
\hline $\begin{array}{l}\text { Lenticulars } \\
\text { NGC } 3384 \\
\text { NGC } 5866 \\
\text { NGC } 7457\end{array}$ & $\begin{array}{l}\mathrm{E} 5 / \mathrm{S} 0 \\
\mathrm{~S} 0 \\
\mathrm{E} 4 / \mathrm{S} 0\end{array}$ & $\begin{array}{l}-19.5 \\
-20.1 \\
-18.6\end{array}$ & $\begin{array}{l}11 \\
14 \\
12\end{array}$ & $\begin{array}{c}\text { CTIO+RFP } \\
\text { WHT+ISIS } \\
\text { WHT+PN.S }\end{array}$ & $\begin{array}{r}50 \\
34 \\
100\end{array}$ & $\begin{array}{c}\text { TW95, SW06 } \\
\text { G00 } \\
\text { in prep }\end{array}$ \\
\hline $\begin{array}{l}\text { Ellipticals } \\
\text { M32 } \\
\text { NGC } 3379 \\
\text { NGC } 1399 \\
\text { NGC } 5128 \\
\text { NGC } 4406 \\
\text { NGC } 1316\end{array}$ & $\begin{array}{l}\text { (early } \\
\text { E } \\
\text { E1 } \\
\text { E1 } \\
\text { E2p } \\
\text { E4 } \\
\text { E3p }\end{array}$ & $\begin{array}{l}\text { work) } \\
-16.4 \\
-19.9 \\
-21.0 \\
-20.7 \\
-21.3 \\
-21.8\end{array}$ & $\begin{array}{c}1 \\
10 \\
19 \\
4 \\
16 \\
20\end{array}$ & $\begin{array}{c}\text { Lick+ITS, KPNO+IIDS } \\
\text { KPNO+NESSIE } \\
\text { NTT+EMMI } \\
\text { AAT+RGO, CTIO+ARGUS } \\
\text { NTT+EMMI } \\
\text { NTT+EMMI }\end{array}$ & $\begin{array}{r}15 \\
29 \\
37 \\
433 \\
19 \\
43\end{array}$ & $\begin{array}{l}\mathrm{NF} 86 \\
\mathrm{C}+93 \\
\mathrm{~A}+94 \\
\mathrm{H}+95 \\
\mathrm{~A}+96 \\
\mathrm{~A}+98\end{array}$ \\
\hline $\begin{array}{l}\text { Ellipticals } \\
\text { NGC } 4697 \\
\text { NGC } 821 \\
\text { NGC } 3379 \\
\text { NGC } 4494 \\
\text { NGC } 5128 \\
\text { NGC } 1344 \\
\text { M } 87 \\
\text { NGC } 4472\end{array}$ & $\begin{array}{c}\text { (recent } \\
\text { E3 } \\
\text { E4 } \\
\text { E1 } \\
\text { E1 } \\
\text { E2p } \\
\text { E5 } \\
\text { E3 } \\
\text { E2 }\end{array}$ & $\begin{array}{l}\text { work) } \\
-19.9 \\
-20.4 \\
-19.9 \\
-20.4 \\
-20.7 \\
-20.2 \\
-21.4 \\
-21.7\end{array}$ & $\begin{array}{c}11 \\
22 \\
10 \\
16 \\
4 \\
19 \\
15 \\
15\end{array}$ & $\begin{array}{c}\text { UT1+FORS1 } \\
\text { WHT+PN.S } \\
\text { WHT+PN.S, CTIO+RFP } \\
\text { WHT+PN.S } \\
\text { AAT+2dF, CTIO+Argus,Hydra } \\
\text { UT3+FORS1 } \\
\text { UT2+FORS2 } \\
\text { WHT+WYFFOS, UT2+FORS2 }\end{array}$ & $\begin{array}{l}535 \\
140 \\
187 \mathrm{R}+ \\
248 \\
780 \\
195 \\
200 \\
80\end{array}$ & $\begin{array}{c}\mathrm{M}+01 \\
\mathrm{R}+03, \text { in prep } \\
+03, \mathrm{SW} 06, \text { in pre } \\
\mathrm{R}+03, \text { in prep } \\
\mathrm{P}+04 \\
\mathrm{~T}+05 \\
\text { in prep } \\
\text { in prep }\end{array}$ \\
\hline
\end{tabular}

The standard observational technique for extragalactic PN kinematics involves an imaging survey with "off-band" and "on-band" images, where "on-band" usually means

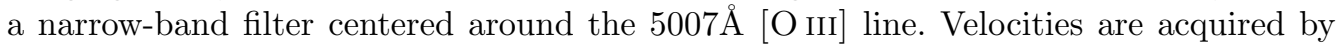
follow-up with a multi-object spectrograph. Some examples of this are wide-field imaging surveys of M 33 and M 83, followed by fiber spectroscopy with velocities accurate to $\sim 5 \mathrm{~km} \mathrm{~s}^{-1}$ (Ciardullo et al. 2004; Herrmann \& Ciardullo 2006).

Another technique is to use a type of slitless spectroscopy called counter-dispersed imaging (CDI), in which PN detection and velocity measurement are done all in one step. Used occasionally with existing instrumentation (e.g., Douglas et al. 2000), CDI has really come into its own with the custom-built Planetary Nebula Spectrograph (PN.S; Douglas et al. 2002). Originally optimized for observation of $5007 \AA$ only, the PN.S has a new arm for simultaneous $\mathrm{H} \alpha$ imaging, due for commissioning in late 2006. This addition allows for improved PN detection reliability, better background contaminant rejection, and the capability of measuring $F([\mathrm{O} \mathrm{III}]) / F(\mathrm{H} \alpha)$ line ratios.

A spectacular success of the PN.S has been the wide-field kinematic survey of the Local Group spiral M 31, with 2615 PN velocities over $7 \mathrm{deg}^{2}$, encompassing much of the bulge, disk, and halo (Merrett et al. 2003, 2006). There is also an extensive traditional imaging+fiber survey (Halliday et al. 2006). Comparing techniques, the PN.S is clearly more efficient, but has slightly worse velocity accuracy, and probably poorer astrometry for facilitating further spectroscopic study. 

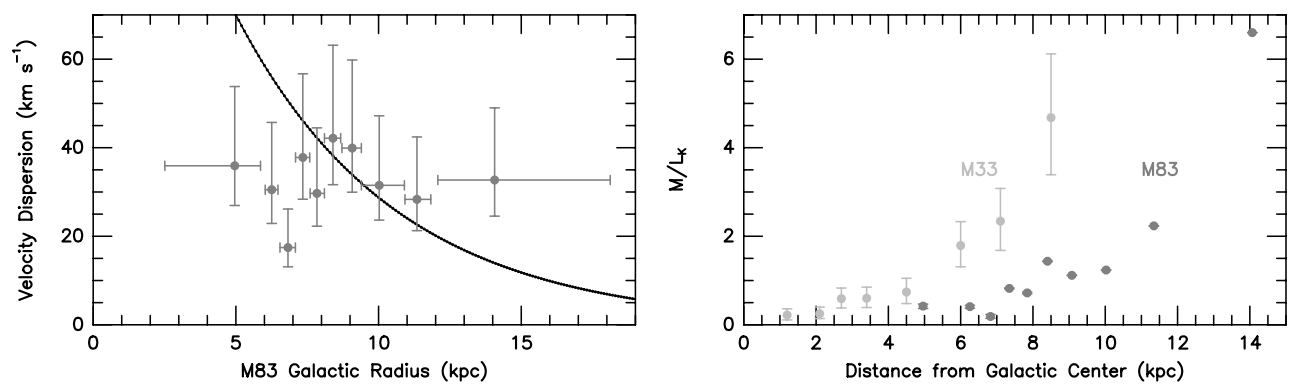

Figure 1. Left: Vertical velocity dispersion profile of $\mathrm{PNe}$ in $\mathrm{M} 83$, as a function of radius. Error-bars show the data, and curve shows expectation from constant mass-to-light ratio disk. Right: Implied local $K$-band mass-to-light ratio of disk, with radius, for M 33 and M 83.

\subsection{Rotation curves of spiral galaxies}

The most fundamental kinematic property of a spiral galaxy is the rotation curve, or circular velocity profile, which provides the cumulative mass profile via the relation $v_{\mathrm{c}}^{2}(r)=G M(r) / r$. It is infeasible to obtain $v_{\mathrm{c}}(r)$ from integrated-light stellar spectroscopy in the nearest spirals because of their large angular extent and rapidly decreasing surface brightness profiles. With PN kinematics in M 31 and M 33, $v_{\mathrm{c}}(r)$ is obtained to 4-6 disk scale-lengths $R_{\mathrm{d}}$, and found to agree with the flat rotation curves obtained from $\mathrm{H}$ I and $\mathrm{CO}$ gas measurements (modulo the asymmetric drift corrections; Ciardullo et al. 2004; Merrett et al. 2006). This appears to rule out magnetic fields as an alternative explanation for the flat rotation curves (e.g., Battaner \& Florido 2005).

\subsection{Disk masses of spiral galaxies}

Although H I measurements in large samples of spiral galaxies make it clear that $v_{\mathrm{c}}(r)$ is fairly constant at large $r$, implying large discrepancies between the visible and total masses (Persic et al. 1996), there is considerable freedom for decomposing $M(r)$ into its subcomponents. Thus there is a strong degeneracy between the mass-to-light ratio $\Upsilon_{*}$ used for the stars, and the inferred properties of the dark matter (DM) halo (e.g., Bottema 1997). The degeneracy can be broken by measuring the velocity dispersion of the stellar disk, especially in the face-on case. This is evident with the isothermal disk approximation, where the vertical velocity dispersion $\sigma_{z}$ is directly proportional to the disk surface mass density $\Sigma$, and the scale-height $z_{0}$ should be fairly constant: $\sigma_{z}^{2}(R)=\pi G \Sigma(R) z_{0}$.

Such dispersions have been measured in inner disks, but one cannot assume that $\Upsilon_{*}$ is the same in the outer disk - and in these low surface brightness regions, PN kinematics are generally the only accessible stellar tracer. In M 33 and M 83, the PN dispersion is found to decrease fairly slowly with radius, implying a disk mass scale-length much larger than the photometric $R_{\mathrm{d}}$ (see Fig. 1, left; Ciardullo et al. 2004; Herrmann \& Ciardullo 2006). This means that $\Upsilon_{*}$ increases markedly with radius (Fig. 1, right) - the opposite to what one expects from inside-out disk formation. A similar effect is seen in M31, where warp models are also tried out, but still don't fit the observations (Merrett et al. 2006).

The M $33 \mathrm{PN}$ measurements imply that the rotation curve is dominated by the disk in the inner parts, apparently ruling out the cuspy dark halo profiles predicted by $\Lambda \mathrm{CDM}$ theory. However, the disk is not massive enough to account for all the "missing mass", ruling out the cold gas DM theory (Pfenniger et al. 1994). Another alternative is the MOND gravitational theory, but with this, the M $33 \mathrm{PN}$ data point to a self-consistency problem (Stubbs \& Garg 2005). It seems the only major model for spiral galaxy mass 
profiles which is not "disproved" is a DM halo with a shallow core-but further work is clearly needed.

\subsection{Lenticular galaxies}

Lenticular galaxies (S0s) are still mysterious objects. It is not known whether they are more akin to spiral galaxies (resulting from gas stripping and quenching), or to ellipticals (resulting from major galaxy mergers). These two basic structural-formational scenarios can be distinguished via their large-radius kinematics - an observational goal we are now beginning to address with the PN.S (E. Noordermeer et al., in prep).

\section{Planetary nebula kinematics in elliptical galaxies}

Elliptical galaxies are notoriously difficult to model, with their complicated internal structures and dearth of suitable kinematic tracers. With PN data, there is the potential for engaging in global "three-dimensional" dynamical studies of ellipticals. For example, on the 4-m William Herschel Telescope, the IFUs SAURON and OASIS (with AO) can probe a galaxy's central parts from parsec to kpc scales, while the PN.S reaches out to tens of kpc. Such contiguous constraints over four decades in radius are important because of the coupling between triaxial orbits in the halo and the central supermassive black hole - though there is an implicit assumption that the PNe and stars share the same dynamics (see $\S 3.3$ ).

\subsection{Observations in elliptical galaxies}

Early work on PNe in elliptical galaxies produced small but tantalizing kinematic samples in their halos (see Table 1). The exception was the nearby NGC 5128, which remains the best-studied early-type galaxy. Recent improvements in instrumentation and methods have led to an explosion of PN velocities (again, see Table 1). Particularly important has been the exploration for the first time of ordinary $L^{*}\left(M_{B} \sim-20.3\right)$ ellipticals' halos.

The panoply of modern observational techniques includes slitless dispersed imaging with the VLT+FORS (Méndez et al. 2001; Teodorescu et al. 2005), whose 535 velocities in NGC 4697 remains by far the largest data-set in an ordinary elliptical. Three ellipticals have been studied with the PN.S as a prelude to a large, systematic sample (Romanowsky et al. 2003). PN kinematics have been measured in the giant Virgo ellipticals M 87 and NGC 4472 (M49) using a "masked-CDI" hybrid technique - useful given pre-imaging data with uncertain astrometry. Fabry-Pérot narrow-band scanning has been used successfully on several galaxies (Sluis \& Williams 2006) - an approach which should become more potent with larger telescopes (e.g., Bershady et al. 2004).

\subsection{Dispersion profiles of elliptical galaxies}

The standard mass indicator in ellipticals is the projected velocity dispersion profile $\sigma_{\mathrm{p}}(R)$, although it is very non-trivial to translate this into $v_{\mathrm{c}}(r)$. In discussing observed $\mathrm{PN}$ dispersions in galaxies, I will also mention the dispersions of globular clusters (GCs) when available, as these are complementary tracers of halo mass.

In NGC $5128, \sigma_{\mathrm{p}}(R)$ is fairly constant at $\sim 120 \mathrm{~km} \mathrm{~s}^{-1}$ for both the PNe and GCs, out to $\sim 10 R_{\text {eff }}$ (effective radii). This implies a dark halo, although perhaps not as massive as expected (Peng et al. 2004). In the brightest cluster galaxy NGC 4472, the dispersion is also flat for the PNe and GCs to $\sim 10 R_{\text {eff }}$ (G. Bergond et al. in prep). In M 87, the elevated PN and GCs dispersion profiles reveal the effects of the massive Virgo Cluster core (Romanowsky \& Kochanek 2001), but their very different slopes imply in this case a difference in the orbital properties between the stars and the GCs. In NGC 1399, the PNe 

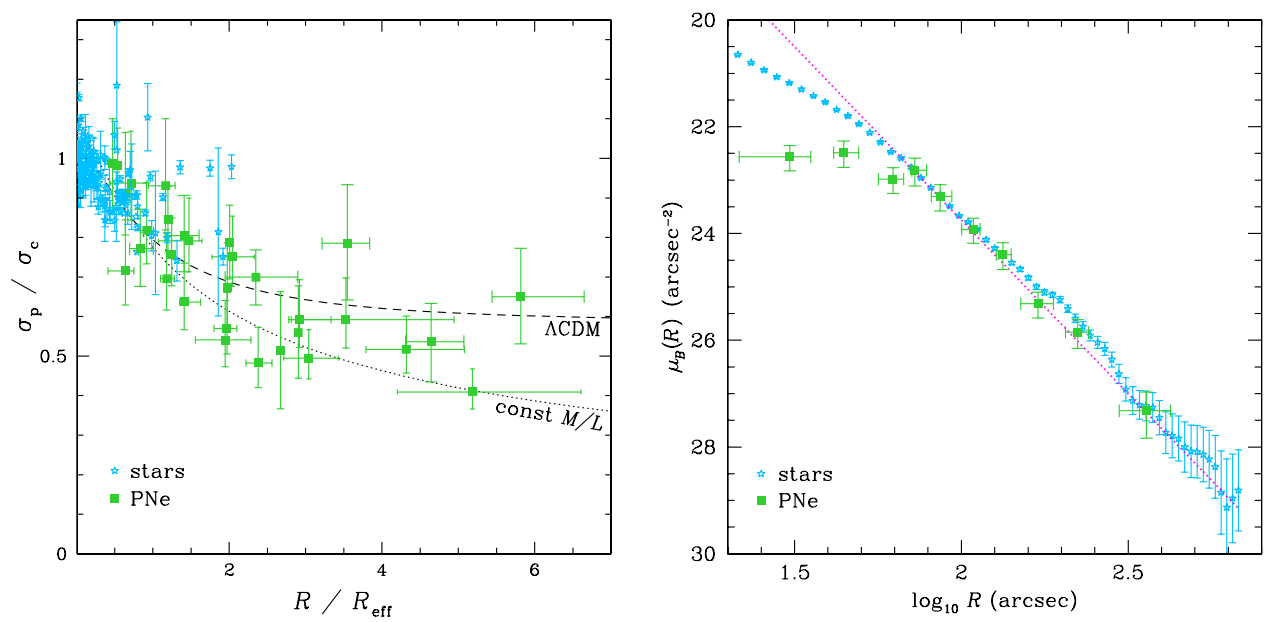

Figure 2. Left: Rescaled and stacked profiles of projected velocity dispersion for 5 ordinary elliptical galaxies. Stellar and PN profiles are shown by different symbols, and simple model predictions are shown by curves. Right: NGC 3379 surface density profiles. Points with error bars show the $B$-band stellar photometry (Capaccioli et al. 1990), and the PN number density (with arbitrary normalization). Inside $75^{\prime \prime}$, the PN data are incomplete. The line shows a steeper density profile that might correspond to young stars formed in a merger (see Dekel et al. 2005).

and GCs also show a rising $\sigma_{\mathrm{p}}(R)$ (Arnaboldi et al. 1994; Y. Schuberth et al. in prep). This may be evidence of the Fornax Cluster core (Saglia et al. 2000), or of transient heating from a galaxy interaction (Napolitano et al. 2002).

More remarkable behavior is seen in the five observed ordinary ellipticals, where $\sigma_{\mathrm{p}}$ declines markedly with radius, with some galaxy-to-galaxy scatter in the slope (Fig. 2, left). This suggests low DM content, as first inferred by Ciardullo et al. (1993) from PN kinematics in NGC 3379. NGC 3379 is the only one of these galaxies so far with GC kinematics data (49 velocities; Puzia et al. 2004; Pierce et al. 2006; Bergond et al. 2006). The GC halo dispersion declines much more gradually than the PNe, but the implication of this is not clear (see $\S 3.4)$.

\subsection{Systematic issues in mass estimation}

Before moving on to direct mass results, I consider a number of complications in deriving elliptical mass profiles from PN kinematics. One is that foreground stars and background emission line sources can masquerade as PNe due to low signal-to-noise or to limited wavelength coverage. With current PN.S data, background emitters are rejected based on resolving the emission in space or wavelength, or on starkly outlying velocities.

The next issue is the well-known mass-anisotropy degeneracy, whereby the types of orbits have an effect on the projected dispersions similar to the impact of the mass distribution. For example, low $\sigma_{p}$ can be caused by low mass or by radially-biased orbits whose motions are largely in the plane of the sky. The model curves shown in Fig. 2 (left) are for isotropic galaxies, but if the $\mathrm{PN}$ orbits in the halo have radial anisotropy, then the $\Lambda \mathrm{CDM}$ prediction could be substantially lowered.

Fortunately, this degeneracy can be broken by examining the shape of the line-ofsight velocity distribution in addition to its width (dispersion). With integrated-light stellar data, this shape is characterized by high-order Gauss-Hermite polynomials, and for discrete PN velocity data, the shape can be inferred statistically. Such analyses can require $\sim 1000$ discrete data points even under the simplifying assumption of sphericity 
(Merritt \& Saha 1993) - but this discouragingly large number is really more relevant for sparse stellar and galaxy clusters. With elliptical galaxies, there are additional constraints that drastically reduce the uncertainties: the PNe are assumed to be drawn from a wellknown spatial distribution, and the stellar kinematics firmly establish the mass and anisotropy characteristics for the central half of the galaxy.

The diffuse stellar component is thus of great importance in exploiting the PN kinematics, which raises the important question of how well do the PNe trace the diffuse stellar light? One effect may arise from radial metallicity gradients, which are thought to exist in the stellar bodies of ellipticals based on their color gradients. A metallicity gradient would imply an oxygen gradient, and thus if the halo PNe had systematically weaker [O III] emission, there would be a bias in the $\mathrm{PN}$ detections resulting in lower observed $\sigma_{\mathrm{p}}$. However, the evidence so far in nearby ellipticals is that halo metallicities have weakly negative or even positive gradients (Gregg et al. 2004; Méndez et al. 2005; Rejkuba et al. 2005; Walsh et al. 2006). Another potential problem is if the PNe are "contaminated" by a young (e.g. < 3 Gyr) population. However, CMD and PN observations find no evidence for young halo populations (Gregg et al. 2004; Peng et al. 2006), and UV fluxes indicate that even in the few early-type galaxies with recent star formation $(<1.5 \mathrm{Gyr})$, this contributes only $1-2 \%$ of the stellar mass (Yi et al. 2005).

A different approach is to directly test the link between the stars and PNe: do they have the same spatial and kinematic distributions in regions of overlap? As mentioned earlier, various studies of PNe in elliptical galaxies have not turned up any indications of population biases. Even in M31, with its spatially-varying star-formation history, there are no evident population effects such as correlations between kinematics and PN luminosity, or variations in the PN luminosity function with radius. In NGC 3379, the surface density and kinematics of the stars and the PNe match up well, although it is difficult to rule out small differences (see Fig. 2, right). The one clear example of a subpopulation signature is in NGC 4697, where an East-West asymmetry of the bright PNe implies an unmixed stellar component-perhaps corresponding to a recent interaction with another galaxy (Sambhus et al. 2006). This feature coincides with the asymmetry of the observational set-up, so confirmation is needed of the result in this galaxy and in others.

Dynamical models usually assume equilibrium, which will not hold in the outermost halo regions where crossing times are long. However, equilibrium should be a good approximation out to $\sim 10 R_{\text {eff }}$ (see Mamon et al. 2006). The final major source of uncertainty is the intrinsic shape of the galaxies. Modeling flattened systems as spherical can skew mass inferences, and in particular, a large face-on disk could produce low velocity dispersions in projection. However, this particular scenario does not work for NGC 821 and NGC 4697, which are near-edge-on "low DM" galaxies.

\subsection{Halo masses of elliptical galaxies}

Having considered all these caveats, we turn to detailed modeling of NGC 3379, with extensive stellar kinematics (Statler \& Smecker-Hane 1999) and an initial data set of $\sim 100$ PN velocities (Romanowsky et al. 2003). An "orbit modeling" method is used which constrains the anisotropy directly from the data, assuming spherical symmetry. The resulting models do indeed have radial anisotropy in the halo, allowing for more DM than suggested by Fig. 2 (left). The $B$-band mass-to-light ratio inside $5 R_{\text {eff }}$ is $7.1 \pm 0.6$ (in Solar units), which is very similar to estimates for the stellar $\Upsilon_{*}$. This result is not what one would expect from $\Lambda$ CDM theory, but given the considerable uncertainties in the mass constraints and in theoretical predictions, $\Lambda$ CDM cannot be ruled out. The other $L^{*}$ galaxies have not yet been modeled in such detail, but Jeans model comparisons suggest similar results, with the exception of NGC 1344. 
While signs of DM from PN kinematics may be skimpy, virial-radius constraints (from weak gravitational lensing, satellite dynamics, and galaxy cross-correlations) do demonstrate the presence of massive halos around ordinary ellipticals. Thus the burning question is not whether ellipticals are "naked" galaxies without DM, but what is the radial distribution of $D M$ that can satisfy all these constraints? This would provide clues about the physics of galaxy formation and about the nature of the DM itself. A meta-analysis of the available kinematic constraints suggests that DM halos in ellipticals have low concentrations (Napolitano et al. 2005), as found in many other studies of late-type galaxies and galaxy clusters. However, these low concentrations may be less of a problem now as the consensus on the cosmological parameter $\sigma_{8}$ is shifting toward much lower values. Perhaps this points toward the use of PNe as precision cosmology tools.

Dekel et al. (2005) argue that far from being a problem for $\Lambda$ CDM, the PN data are a natural outcome. Simulating the formation of elliptical galaxies via spiral mergers, they find familiarly declining $\sigma_{p}(R)$ profiles. The physics of the simulations may not adequately represent the formation of galaxies in $\Lambda \mathrm{CDM}$, but in any case, the study highlights three major effects that could contribute to the declines. The first is varying radial anisotropy, which indeed is included in our direct modeling of NGC 3379. The second is that the triaxial structure of the galaxies causes variations in $\sigma_{\mathrm{p}}$ with the viewing angles. The third is that if the observed PNe stem from a young stellar population formed in the merger, then they are not a fair tracer of the bulk of the stars (see $\S 3.3$ ), and produce a steeper decline in $\sigma_{\mathrm{p}}$.

More definitive statements on PN-based mass profiles obviously await experiments with triaxial models (e.g., De Lorenzi et al. 2006). The large PN.S program sample will also be able to average out viewing-angle effects. Of further use are independent mass constraints, such as GCs and X-ray emission. As mentioned, in NGC $3379, \sigma_{\mathrm{p}}(R)$ is very different for the PNe and GCs, which can be largely explained through different spatial and anisotropy characteristics. The GCs do suggest a more massive halo, but this appears to be contradicted by the kinematics of a large-radius $\mathrm{H}$ I gas ring implying a remarkably low-mass halo (Schneider 1985).

\section{Summary}

Planetary nebula are powerful tools for exploring galaxy mass profiles, and with the advent of new instrumentation, the sample of galaxies with PN kinematics is now exploding. In spiral galaxies, fairly constant $\mathrm{PN}$ velocity dispersions imply surprisingly large disk mass scale-lengths. In ordinary ellipticals, declining PN dispersions suggest low-concentration dark matter halos. An important issue still needing clarification for dynamical models is the connection between PNe and their parent stellar population.

\section{Acknowledgements}

I would like to thank Kim Herrmann \& Robin Ciardullo for providing their plots, the Scientific Organizing Committee for their invitation, and the IAU for travel support.

\section{References}

Arnaboldi, M., Freeman, K.C., Hui, X., et al. 1994, The Messenger, 76, 40 (A+94)

Arnaboldi, M., Freeman, K.C., Mendez, R.H., Capaccioli, M., et al. 1996, ApJ, 472, 145 (A+96)

Arnaboldi, M., Freeman, K.C., Gerhard, O., Matthias, M., et al. 1998, ApJ, 507, 759 (A+98)

Battaner, E. \& Florido, E. 2005, (astro-ph/0503657)

Beaulieu, S.F., Freeman, K.C., Kalnajs, A.J., Saha, P. \& Zhao, H. 2000, AJ, 120, 855 
Bergond, G., Zepf, S.E., Romanowsky, A.J., Sharples, R.M., Rhode, K.L. 2006, A $\& A$, 448, 155 Bershady, M.A., Verheijen, M.A.W., Andersen, D.R., Swaters, R.A. \& Westfall, K.B. 2004, in: D.A.H. Buckley (ed.), The First Robert Stobie SALT Workshop (astro-ph/0403478)

Bottema, R. 1997, A\&A, 328, 517

Buzzoni, A., Arnaboldi, A. \& Corradi, R.L.M. 2006, MNRAS, 368, 877

Capaccioli, M., Held, E.V., Lorenz, H. \& Vietri, M. 1990, AJ, 99, 1813

Ciardullo, R., Durrell, P.R., Laychak, M.B., Herrmann, K.A., et al. 2004, ApJ, 614, 167 (C+04)

Ciardullo, R., Jacoby, G.H. \& Dejonghe, H.B. 1993, ApJ, 414, 454 (C+93)

Ciardullo, R., Sigurdsson, S., Feldmeier, J.J. \& Jacoby, G.H. 2005, ApJ, 629, 499

Dekel, A., Stoehr, F., Mamon, G.A., Cox, T.J., et al. 2005, Nature, 437, 707

De Lorenzi, F., Debattista, V.P. \& Gerhard, O.E. 2006, in: L. Stanghellini, J.R. Walsh \& N.G. Douglas (eds.), Planetary Nebulae Beyond the Milky Way (Berlin: Springer), p. 311

Douglas, N.G., Gerssen, J., Kuijken, K. \& Merrifield, M.R. 2000, MNRAS, 316, 795 (D+00)

Douglas, N.G., Arnaboldi, M., Freeman, K.C., Kuijken, K., et al. 2002, PASP, 114, 1234

Gerssen, J. 2000, PhD thesis, Rijksuniversiteit Groningen (G00)

Gregg, M.D., Ferguson, H.C., Minniti, D., Tanvir, N. \& Catchpole, R. 2004, AJ, 127, 1441

Halliday, C., Carter, D., Bridges, T.J., et al. 2006, MNRAS, 369, $97(\mathrm{H}+06)$

Herrmann, K.A. \& Ciardullo, R., 2006, in prep (HC06)

Hui, X., Ford, H.C., Freeman, K.C. \& Dopita, M.A. 1995, ApJ, 449, 592 (H+95)

Maciel, W.J. \& Lago, L.G. 2005, Rev. Mexicana AyA, 41, 383

Mamon, G.A., Łokas, E., Dekel, A., et al. 2006, in: G.A. Mamon, et al. (eds.), Mass Profiles and Shapes of Cosmological Structures (Paris: EDP), p. 139 (astro-ph/0601345)

Marigo, P., Girardi, L., Weiss, A., Groenewegen, M.A.T. \& Chiosi, C. 2004, A\&A, 423, 995

Méndez, R.H., Riffeser, A., Kudritzki, R.-P., Matthias, M., et al. 2001, ApJ, 563, 135 (M+01)

Méndez, R.H., Thomas, D., Saglia, R.P., Maraston, C., et al. 2005, ApJ, 627, 767

Merrett, H.R., Kuijken, K., Merrifield, M.R., Romanowsky, A.J., et al. 2003, MNRAS, 346, L62

Merrett, H.R., Merrifield, M.R., et al. 2006, MNRAS, 369, 120 (M+06)

Merritt, D. \& Saha, P. 1993, ApJ, 409, 75

Napolitano, N.R., Arnaboldi, M. \& Capaccioli, M. 2002, A\&A, 383, 791

Napolitano, N.R., Capaccioli, M., Romanowsky, A.J., et al. 2005, MNRAS, 357, 691

Nolthenius, R. \& Ford, H. 1986, ApJ, 305, 600 (NF86)

Peng, E.W., Ford, H.C. \& Freeman, K.C. 2004, ApJ, 602, $685(\mathrm{P}+04)$

Peng, E.W., Ford, H.C. \& Freeman, K.C. 2006, in: L. Stanghellini, et al. (eds.), Planetary Nebulae Beyond the Milky Way (Berlin: Springer), p. 59 (astro-ph/0412449)

Persic, M., Salucci, P. \& Stel, F. 1996, MNRAS, 281, 27

Phillips, J.P. 2001, A\&\&A, 367, 967

Pierce, M., Beasley, M.A., Forbes, D.A., Proctor, R., et al. 2006, MNRAS, 366, 1253

Pfenniger, D., Combes, F. \& Martinet, L. 1994, A\&A A, 285, 79

Puzia, T.H., Kissler-Patig, M., Thomas, D., Maraston, C., et al. 2004, A\&A, 415, 123

Rejkuba, M., Greggio, L., Harris, W.E., Harris, G.L.H., \& Peng, E.W. 2005, ApJ, 631, 262

Romanowsky, A.J., Douglas, N.G., Arnaboldi, M., Kuijken, K., et al. 2003, Science, 301, 1696 $(\mathrm{R}+03)$

Romanowsky, A.J. \& Kochanek, C.S. 2001, ApJ, 553, 722

Saglia, R.P., Kronawitter, A., Gerhard, O. \& Bender, R. 2000, AJ, 119, 153

Sambhus, N., Gerhard, O. \& Méndez, R.H. 2006, AJ, 131, 837

Schneider, S.E. 1985, ApJ (Letters), 288, L33

Schneider, S.E. \& Terzian, Y. 1983, ApJ (Letters), 274, L61

Sluis, A.P.N. \& Williams, T.B. 2006, AJ, 131, 2089 (SW06)

Statler, T.S. \& Smecker-Hane, T. 1999, AJ, 117, 839

Stubbs, C.W. \& Garg, A. 2005, (astro-ph/0512067)

Teodorescu, A.M., Méndez, R.H., Saglia, R.P., Riffeser, A., et al. 2005, ApJ, 635, 290 (T+05)

Tremblay, B., Merritt, D. \& Williams, T.B. 1995, ApJ (Letters), 443, L5 (TW95)

Walsh, J.R., Jacoby, G.H., Peletier, R. \& Walton, N.A. 2006, in: L. Stanghellini, J.R. Walsh \& N.G. Douglas (eds.), Planetary Nebulae Beyond the Milky Way (Berlin: Springer), p. 262

Yi, S.K., Yoon, S.-J., Kaviraj, S., Deharveng, J.-M., et al. 2005, ApJ (Letters), 619, L111 